\title{
Analysis of channel interpolation algorithms ror mobile wimax standard
}

\author{
Nguyen Duc Quang ${ }^{1}$, Luu Thanh Tra ${ }^{1}$, Pham Hong Lien ${ }^{2}$
${ }^{1}$ University of Technology, Vietnam National University, Ho Chi Minh city
Email:quangnd@vms.com.vn, luu@ $@$ hcmut.edu.vn \\ ${ }^{2}$ University of Technical Education, Ho Chi Minh City \\ Email: phamhonglien2005@yahoo.com
}

Abstract: The fourth generation (4G) wireless products are expected to be based on worldwide interoperability for microwave access (WiMAX) systems. In this paper, the issue of channel estimation in the mobile WiMAX systems (IEEE 802.16e-2005) is analyzed. We study by simulation the use of the least square channel estimation algorithm combining with several modulation schemes and interpolation algorithms. The aim is to choose the most feasible interpolation algorithms for each modulation scheme of mobile WiMAX. The results show that the linear and the cubic sub-division interpolations perform the best and they are proposed to be used in modulation schemes.

Keywords: WiMAX, estimation channel, interpolation algorithms.

\section{INTRODUCTION}

In the last few years, mobile communication in Vietnam has shown a dramatically fast growth and it becomes one of the mostly used communication method between people. The biggest Vietnam mobile operators such as Mobifone, Vinaphone and Viettel are now exploiting $3 \mathrm{G}$ technologies while $4 \mathrm{G}$ are being tested and investigated for future deployment. One of the popular $4 \mathrm{G}$ technologies is mobile WiMAX, which is based on IEEE 802.16e standard.

WiMAX is a new technology in the wireless community. It enables operators to offer diverse wireless services to fixed and mobile users. Orthogonal Frequency Division Multiplexing (OFDM) is used for fixed users (IEEE-802.16d-2004) and Orthogonal Frequency Division Multiple Access (OFDMA) transmission techniques are employed for mobile users (IEEE-802.16e-2005) [1].

Mobile WiMAX integrates many modern technologies such as MIMO (multi input multi output), OFDMA (multi-user Orthogonal Frequency Division Multiplexing), AMC (Adaptive Modulation and Coding).

The OFDM has been chosen to be used in WiMAX since it shows many advantages. OFDM is a multicarrier modulation scheme in which the transmission bandwidth is divided into several narrower bands and the data is transmitted in parallel on these bands. As the result, the symbol period is increased by the number of bands, decreasing the effect of intersymbol interference (ISI). The remaining ISI effect is eliminated by extending the signal cyclically. With these properties, OFDM can satisfy the increasing requirements for data in future wireless applications [2].

WiMAX provides many advantages to operators as well as wireless users. The allocation of spectrum in licensed/unlicensed bands gives the operators a chance of service deployment worldwide.

The receiver's algorithms are among the main solutions contributing to the advantages of WiMAX systems. This article focuses on the channel estimation, one of the key blocks in the WiMAX receivers. The channel estimation has been studied extensively for OFDM based systems. The approaches based on least squares (LS) and minimum meansquare error estimation (MMSE) [3] are proposed by 
Research, Development and Application on Information and Communication Technology

inserting the training sequences which are transmitted along with the data. In [4], LS algorithm with linear interpolation and Frequency Averaging Time Interpolation (FATI) is analyzed for mobile WiMAX channel estimation. These algorithms are low order interpolation and have low computational complexity. In addition, other algorithms such as LMMSE, adaptive estimation, are considered in [5]. The estimator, which can utilize the information from one dimension - either time or frequency, is referred to as

1D estimator. If both time and frequency information are utilized the estimator is called 2D estimator.

Although many such channel estimation techniques are reported in the literature, for practical WiMAX systems, it is important to have an estimation technique which is specifically designed for WiMAX preambles or pilots, having low computational and hardware complexities, to be applicable in the real environment.

For that reason, in this paper we select the estimator with the lowest computation complexity the LS (least square) estimator. We simulate this algorithm under different modulation schemes for mobile WiMAX standard and under different channel interpolation algorithms from low order to high order. We have built an adaptive LS algorithm, which can change the interpolation algorithms and modulation schemes depending on the SNR condition. This paper analyzes interpolation algorithms under mobile WiMAX parameters so that we can have the best solutions in each modulation scheme.

The rest of the paper is organized as follows. In Section II, system model is introduced and investigated. The channel interpolation algorithms are detailed in Section III. Section IV describes the mobile WiMAX parameters. Simulation results are presented in Section V. Section VI is the conclusion.

\section{SYSTEM MODEL}

OFDM converts serial data stream into parallel blocks of size $N$ and modulates these blocks using Inverse Fast Fourier Transform (IFFT). Time domain samples of an OFDM symbol can be obtained from frequency domain data symbols. Figure 1 shows a typical block diagram of OFDM system with pilot signal assisted. The binary data are grouped and mapped into multi-amplitude-multi-phase signals.

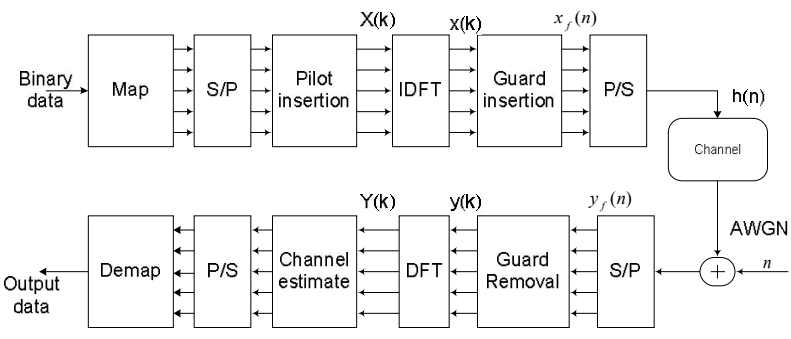

Figure 1. OFDM system structure

In this paper, we consider 3 modulation schemes: 4-QAM (QPSK), 16-QAM, 64-QAM modulation. After the insertion of the pilot signal, the modulated data $\{X(k)\}$ are sent to an IDFT, transformed and multiplexed into $\{x(n)\}$ :

$$
\begin{aligned}
x(n) & =\operatorname{IDFT}\{X(k)\}, n=0,1,2, \ldots, N-1 \\
& =\sum_{k=0}^{N-1} X(k) e^{j(2 \pi k n / N)},
\end{aligned}
$$

where $N$ is the number of sub-carriers. The guard interval is also inserted to prevent possible intersymbol interference in the OFDM systems, resulting:

$$
x_{f}(n)=\left\{\begin{array}{lll}
x(N+n) & , & n=-N_{g},-N_{g}+1, \ldots,-1 \\
x(n) & , & n=0,1, \ldots, N-1
\end{array}\right.
$$

where $N_{g}$ is the number of samples in the guard interval. The transmitted signal is then sent to a frequency selective multi-path fading channel. The received signal can be represented by

$$
y_{f}(n)=x_{f}(n) \otimes h(n)+w(n)
$$

where $h(n)$ is the impulse response of channel and $w(n)$ is the additive white Gaussian noise. The channel impulse responding $h(n)$ can be expressed as follow: 


$$
h(n)=\sum_{l=0}^{L-1} h_{l} e^{j(2 \pi / N) f_{D_{l}} T n} \delta\left(\tau-\tau_{l}\right) ; n=0,1, \ldots . N-1
$$

where:

$L:$ Channel impulse response length.

$h_{l}$ : the complex impulse response of the $1^{\text {th }}$ path.

$f_{D_{l}}$ : the $1^{\text {th }}$-path Doppler frequency shift at the $1^{\text {th }}$ path $\tau$. the delay spread index.

$T$ : sampling time.

$\tau_{i}$ : the $1^{\text {th }}$-path delay time normalized by sampling time in frequency domain, i.e.,

$$
\mathrm{Y}(\mathrm{k})=\operatorname{DFT}\{\mathrm{y}(\mathrm{n})\}=\sum_{n=0}^{N-1} y(n) e^{-j(2 \pi k n / N)}
$$

Suppose that the guard interval is longer than the length of channel impulse response (that is, there is no inter-symbol interference between OFDM symbols), from $\{Y(k)\}$ and the channel transfer function $\{H(k)\}$ the transmitted signal is estimated as below:

$$
X_{e}=\frac{Y(k)}{H_{e}(k)}, \mathrm{k}=0,1, \ldots \mathrm{N}-1
$$

where $H_{e}(k)$ is an estimation of $H(k)$. This estimation of the transmitted signal is called LS (least square). This algorithm does not need to know any knowledge of the statistics of the channels, hence it has very low complexity, but they suffer from a high mean-square error [6].

Based on the principle of OFDM transmission scheme, it is easy to assign the pilot both in time- and frequency domain. Several types of pilot arrangement have been studied. The comb-type pilot [7] (Figure $2 b)$ arrangement is used in this paper.

Figure 2 shows two major types of pilot arrangement. The first kind of pilot arrangement (Figure 2a) is referred as block-type pilot arrangement. The pilot signal is assigned to a particular OFDM block, which is sent periodically in time-domain. The second kind of pilot arrangement (Figure 2b) is called comb-type pilot arrangement. These pilot signals are uniformly distributed within each OFDM block. Assuming that the payloads of pilot signals of the two arrangements are the same, the comb-type pilot assignment has a higher retransmission rate. Thus, the comb-type pilot arrangement system provides better resistance to fastfading channels [8]. Since only some sub-carriers contain the pilot signal, the channel response of nonpilot sub-carriers will be estimated by interpolating neighboring pilot sub-channels. The analysis of the channel interpolation algorithms are presented in the next section.
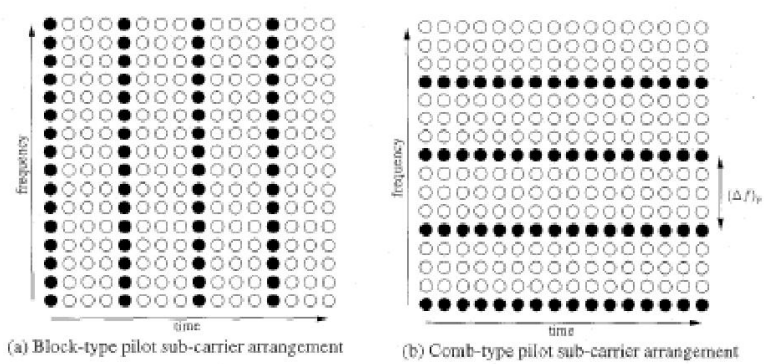

\section{Figure 2. Two different types of pilot sub-carrier arrangement}

\section{CHANNEL INTERPOLATION ALGORITHMS}

In channel estimation, the use of effective channel interpolation algorithm is necessary to estimate channel at the sub-carrier frequency by using the information from comb-type pilot. This section analyzes several interpolation algorithms which are used in channel estimation.

\section{A. The nearest neighbor interpolation algorithm (NNI)}

This method is very simple by choosing the value of the nearest point to be the interpolated point. The algorithm just calculates the distances between interpolated point and the surrounding points and selects the one with minimum distance. The algorithm is simple, easy to execute and it is very popular in image processing. This algorithm looks like order zero of interpolation

\section{Example 1:}

Given $\mathrm{x}=\left[\begin{array}{llll}0 & 4 & 8 & 12\end{array}\right]$ and corresponding $\mathrm{y}=$ 
Research, Development and Application on Information and Communication Technology

$y=\left[\begin{array}{llll}2 & 10 & 6 & 20\end{array}\right]$. We interpolate another points $(1,2,3$,

$5,6,7, \ldots)$. Below is the interpolated result:

$\mathrm{y}_{\mathrm{NNI}}=\left[\begin{array}{lllllllllllll}2 & 2 & 10 & 10 & 10 & 10 & 6 & 6 & 6 & 6 & 20 & 20 & 20\end{array}\right]$.

The above given condition will be used later in other interpolations to see difference between them.

\section{B. Linear interpolation algorithm (LI)}

In the linear interpolation algorithm, two successive pilot sub-carriers are used to determine the channel response for data sub-carriers that are located in between the pilots. For data sub-carrier $k, m L<k<$ $(m+l) L$, the estimated channel response using linear interpolation method is given by:

$$
\begin{aligned}
& H_{e}(k)=H_{e}(m L+l) \quad 0 \leq l<L \\
= & \left(H_{p}(m+1)-H_{p}(m)\right) \frac{l}{L}+H_{p}(m)
\end{aligned}
$$

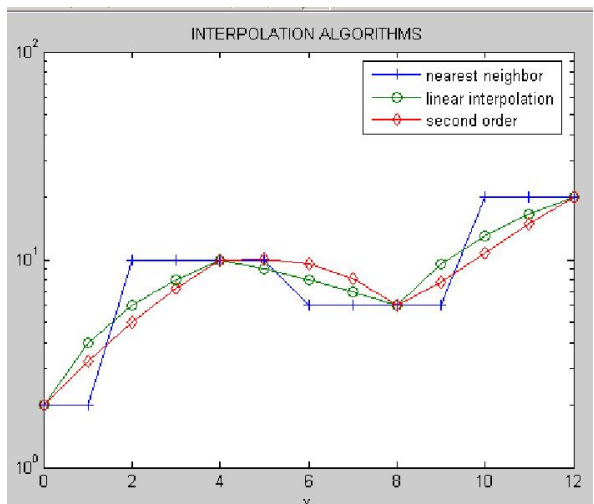

Figure 3. Comparison between different interpolation algorithms

This algorithm is the first order of interpolation. Note that the nearest neighbor interpolation and the linear interpolation are also known as low order interpolation algorithms because their simplicity.

Applying this interpolation to the previous example, we have:

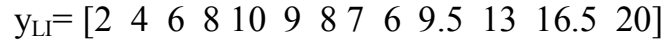

C. The second order polynomial interpolation algorithm (SOPI)

The second order interpolation determines the interpolated point based on three successive points, given by

$$
\begin{gathered}
H_{e}(k)=H_{e}(m L+l)=c_{1} H_{p}(m-1)+c_{0} H_{P}(m)+c_{-1} H_{P}(m+1) \\
\left\{\begin{array}{l}
c_{1}=\frac{\alpha(\alpha-1)}{2} \\
c_{0}=-(\alpha-1)(\alpha+1), \alpha=\frac{l}{L} \\
c_{-1}=\frac{\alpha(\alpha+1)}{2}
\end{array}\right.
\end{gathered}
$$

Theoretically, using higher-order polynomial interpolation will fit the channel response better than the linear interpolation. However, the computational complexity grows as the order is increased. Here we consider the second-order polynomial interpolation for its acceptable computational complexity.

The result of this interpolation applying to the example is:

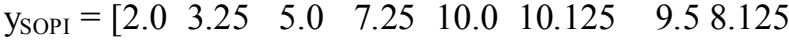

$\left.\begin{array}{lllll}6.0 & 7.8125 & 10.75 & 14.8125 & 20.0\end{array}\right]$

\section{Low pass interpolation (LPI)}

Low pass interpolation can be given as below: insert zero at sub carrier frequency then design a symmetric FIR filter with the condition that the original data are passed without any change and determine interpolated points with condition that MSE (mean square error) between interpolated points and pilot point is smallest.

Again, with the Example 1, we have the result of interpolation: $\mathrm{y}_{\mathrm{LPI}}=\left[\begin{array}{lllll}2.0 & 4.353 & 6.6013 & 8.5422 & 10.0\end{array}\right.$ $\begin{array}{llllll}9.6441 & 8.8017 & 7.5495 & 6.0 & 10.3043 & 14.3028\end{array}$ $17.6353 \quad 20.0]$

\section{E. Spline cubic interpolation algorithm (SCI)}

This algorithm makes the interpolated points to be continuous and smooth with original data. The idea of this algorithm is to replace the $n$ order polynomial by 
the lower order polynomial such as order $1,2,3 \ldots$ on each range $\left[x_{k} x_{k+1}\right], k=0 \ldots n-1$ and spline cubic mean the polynomial used to interpolate is the third order

With Example 1, we have;

$$
\mathrm{y}_{\mathrm{SCI}}=\left[\begin{array}{lllll}
2.0 & 6.7656 & 9.375 & 10.2969 & 10.0
\end{array}\right.
$$

$\begin{array}{lllllll}8.9531 & 7.6250 & 6.4844 & 6.0 & 6.6406 & 8.8750\end{array}$ $13.171920 .0]$

F. Cubic sub-division interpolation algorithm

(CSDI)

Given a control polygon $P=\left\{P_{0}, P_{l}, \ldots, P_{n}\right\}$ we generate a refinement of $P$ by generating a new control polygon

$\left\{E_{0}, V_{0}, E_{1}, V_{1}, E_{2}, \ldots, E_{n-2}, V_{n-2}, E_{n-1}\right\}$

where $E_{i}$, the edge points, lie on the edges of the original control polygon, and $V_{i}$, the vertex points, correspond to the original vertices of the control polygon. The new control polygon has $2 n-1$ control points (the original has $n+1$ ) - and thus each refinement step increases the number of points in the control polygon. The rules are to calculate the vertex and edge points from the subdivision process for a uniform B spline curve. In short,

$$
E_{i}=\frac{P_{i}+P_{i+1}}{2}
$$

and

$$
V_{i}=\frac{E_{i}+2 P_{i}+E_{i+1}}{4}
$$

Figure 4 demonstrates this algorithm: from the first points $P=\left\{P_{1}, P_{2}, P_{3}, P_{4}, P_{5}\right\}$, we can interpolate other points: $E_{0}, E_{1} \ldots$ then continue with the interpolating points: $V_{0}, V_{l}, V_{2}, \ldots$ This process is repeated.

The result of CSDI applied for Example 1 is following:

$\mathrm{y}_{\mathrm{CSDI}}=\left[\begin{array}{llllll}2.0 & 2.9063 & 5.125 & 7.9063 & 10.0 & 9.2188\end{array}\right.$ $\begin{array}{llllllll}7.6250 & 6.2422 & 6.0 & 8.8203 & 12.75 & 16.7031 & 20.0] .\end{array}$

Figure 5 summarizes the results of the discussed interpolation algorithms.

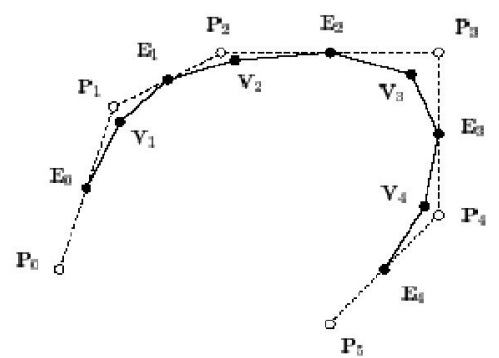

Figure 4. Cubic sub-division algorithm

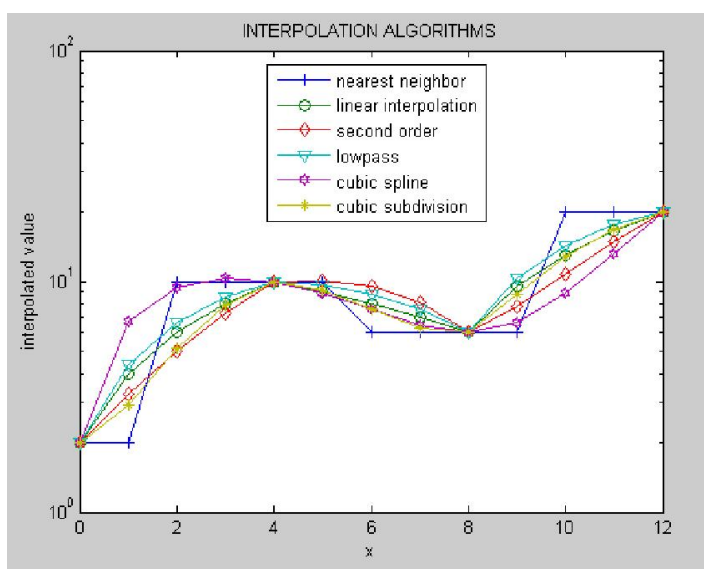

Figure 5. Comparison between different interpolation algorithms

\section{MOBILE WIMAX PARAMETERS}

\section{A. ITU-R channel model}

The channel models can be classified into two main categories: statistical and empirical models. Empirical models are based on measurements performed in real environments, while the statistical models estimate the channel's characteristics through mathematical relations. Two empirical models are generally used for describing a WiMAX system: the SUI (Stanford University Interim) channel model, which is used for simulating an IEEE 802.16-2004 system (fixed WiMAX system) [9] and the ITU-R channel model, which was developed according to the ITU-R M.1225 Recommendation. This type of model is used for the simulation of an IEEE 80216e-2005 system (mobile WiMAX system). 
Research, Development and Application on Information and Communication Technology

Initially developed for the IMT-2000 technologies, the ITU-R channel model is used for modeling a mobile WiMAX system, since mobile WiMAX was adopted by ITU as one of the IMT-2000 technologies in November 2007. The ITU wideband channel, is described based on a tapped delay line model, with a maximum number of 6 taps [10].

ITU's channel model for indoor, pedestrian and vehicular environments is presented as following and empirical model (channel B) is used to describe multipath channel

\section{Table 1. Indoor channel model}

\begin{tabular}{|c|c|c|c|c|}
\hline \multirow{2}{*}{ Tap } & \multicolumn{2}{|c|}{ Channel A } & \multicolumn{2}{c|}{ Channel B } \\
\cline { 2 - 5 } & Delay(ns) & Power (dB) & Delay (ns) & Power (dB) \\
\hline $\mathbf{1}$ & 0 & 0 & 0 & 0 \\
\hline $\mathbf{2}$ & 50 & -3 & 100 & -3.6 \\
\hline $\mathbf{3}$ & 110 & -10 & 200 & -7.2 \\
\hline $\mathbf{4}$ & 170 & -18 & 300 & -10.8 \\
\hline $\mathbf{5}$ & 290 & -16 & 500 & -18 \\
\hline $\mathbf{6}$ & 310 & -32 & 700 & -25.2 \\
\hline
\end{tabular}

Table.2. Pedestrian channel model

\begin{tabular}{|c|c|c|c|c|}
\hline \multirow{2}{*}{ Tap } & \multicolumn{2}{|c|}{ Channel A } & \multicolumn{2}{c|}{ Channel B } \\
\cline { 2 - 5 } & Delay(ns) & Power (dB) & Delay(ns) & Power (dB) \\
\hline $\mathbf{1}$ & 0 & 0 & 0 & 0 \\
\hline $\mathbf{2}$ & 110 & -9.7 & 200 & -0.9 \\
\hline $\mathbf{3}$ & 190 & -19.2 & 800 & -4.9 \\
\hline $\mathbf{4}$ & 410 & -22.8 & 1200 & -8 \\
\hline $\mathbf{5}$ & - & - & 2300 & -7.8 \\
\hline $\mathbf{6}$ & - & - & 3700 & -23.9 \\
\hline
\end{tabular}

Table 3 Vehicular channel model

\begin{tabular}{|c|c|c|c|c|}
\hline \multirow{2}{*}{ Tap } & \multicolumn{2}{|c|}{ Channel A } & \multicolumn{2}{c|}{ Channel B } \\
\cline { 2 - 5 } & Delay(ns) & Power (dB) & Delay (ns) & Power (dB) \\
\hline $\mathbf{1}$ & 0 & 0 & 0 & 0 \\
\hline $\mathbf{2}$ & 0.4 & -1 & 0.8 & -1 \\
\hline $\mathbf{3}$ & 0.8 & -9 & 1.6 & -9 \\
\hline $\mathbf{4}$ & 1.2 & -10 & 2.2 & -10 \\
\hline $\mathbf{5}$ & 1.8 & -15 & 3.6 & -15 \\
\hline $\mathbf{6}$ & 2.6 & -20 & 5.2 & -20 \\
\hline
\end{tabular}

Along with the multi-path problem, the channel model is still effected by the Gaussian noise. Hence, channel interpolation is difficult without studying channel, this paper analyzes the channel interpolation algorithms that can be used in channel estimation and find out the best solution to interpolate the radio channel.

\section{B. Parameters used in the paper}

The paper uses parameters as below, following standard IEEE $802.16 \mathrm{E}$ to simulate mobile WiMAX system.

Table 4. IEEE 802.16E parameters used in the paper

\begin{tabular}{|c|c|l|}
\hline Parameter & Used Value & Notes \\
\hline $\mathrm{N}$ & 1024 & FFT size \\
\hline $\mathrm{N}_{\mathrm{u}}$ & 840 & Number of used subcarriers \\
\hline $\mathrm{N}_{\mathrm{CP}}$ & 256 & CP size \\
\hline $\mathrm{N}_{\mathrm{P}}$ & 280 & $\begin{array}{l}\text { Number of used (pilot) } \\
\text { subcarriers in the preamble }\end{array}$ \\
\hline
\end{tabular}

We simulate the system at the $2.5 \mathrm{GHz}$ with bandwidth $20 \mathrm{MHz}$ and indoor, pedestrian and vehicular models use mobile speed at $1 \mathrm{~km} / \mathrm{h}, 5 \mathrm{~km} / \mathrm{h}$ $100 \mathrm{~km} / \mathrm{h}$ respectively.

At the physical layer of standard IEEE $802.16 \mathrm{E}$, adaptive modulation is used with modulation schemes such as QPSK (4-QAM), 16-QAM and 64-QAM that depend on environment conditions. In this paper, we use 3 modulation schemes that are 4-QAM, 16-QAM and 64-QAM to analyze channel interpolation algorithms.

The channel is effected by multi-path problem of Fading Rayleigh channel and Gaussian noise. The channel is simulated by using channel B of ITU-R channel.

We transmit data at transmitter with pilot insertion. The pilot training has comb structure. At the receiver, we base on pilot information from pilot data extractor to use LS (least square) for estimating the channel at pilot sub-carriers. There are 840 data sub-carriers and 280 pilot sub-carriers. After estimating channel at pilot sub-carriers, we use interpolation algorithms to interpolate channel at positions of data sub-carriers. In 
next section, we simulate and analyze many interpolation algorithms used in channel estimation.

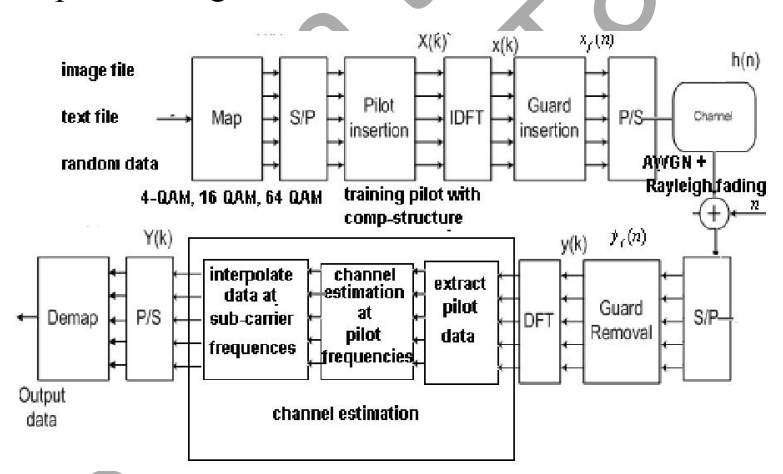

Figure 6. Channel estimation model in simulation

Figure 7 is the estimation channel model we use in this paper. Input file can be text, image file or any random data. At the transmitter, pilot and Guard insertion are done and the information is extracted at the receiver. Radio channels are effected by Gaussian noise and Rayleigh fading in ITU channel B model. Estimation process is done at the receiver. After extracting pilot data, channel is estimated at pilot frequencies. Then, we use interpolation algorithms to interpolate data at sub-carrier frequencies.

\section{SIMULATIONS AND RESULTS:}

\section{A. No channel estimation:}

To see what is effected by Gaussian noise and fading, we transmit an image file at transmitter. Then the data is effected by Gaussian noise and Rayleigh fading. At the receiver, demodulation process is run to extract original data. Many cases are analyzed to compare between eachother with or without estimation channel.

Firstly, one of channel effects we can see easily is power of the receiver. With higher power receiver, we have a stronger signal and there are less error bits at the receiver.

In the following simulations, 4-QAM modulation is used, we can see Gaussian noise does not impact on data with $\mathrm{SNR}=20 \mathrm{~dB}$ and there are no error bit at the receiver with $\mathrm{SNR}=20 \mathrm{~dB}$. However, with $\mathrm{SNR}=5 \mathrm{~dB}$, there are a lot of error bits.

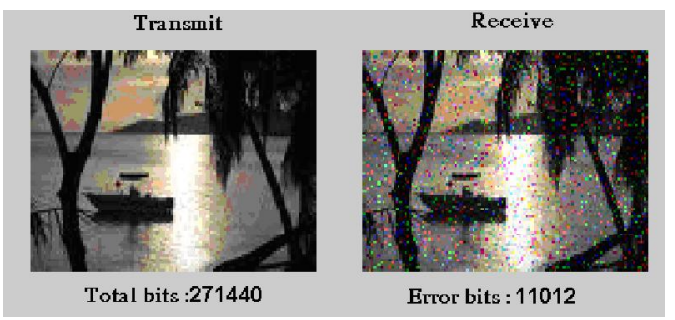

Figure 7. Simulation without channel estimation, $S N R=5 d B, 4-Q A M$ with only Gaussian noise impact

Secondly, another impact we should know is the order of modulation scheme. With higher order simulation scheme, we have a better performance. However, the distance between two symbols with higher order simulation, is closer. Hence, noise makes impact on the channel easier and there will be error bits. We can find in the following simulation, with the same data at Figure there are error bits when 64-QAM modulation is used while in Figure 9 see any error bit.

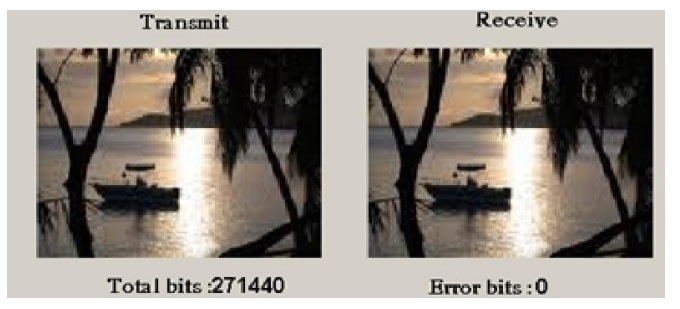

Figure 8. Simulation without channel estimation, $S N R=20 \mathrm{~dB}, 4-Q A M$ with only Gaussian noise impact

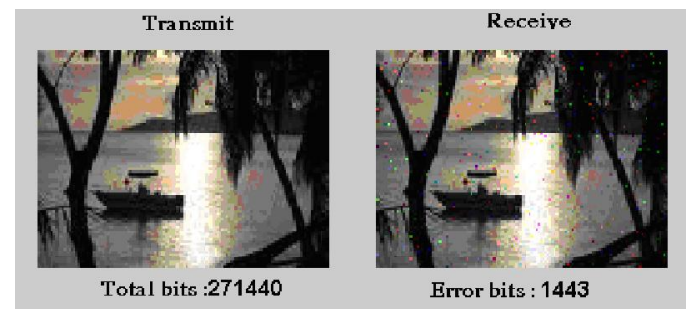

Figure 9. Simulation without channel estimation, $S N R=20 d B, 64-Q A M$ with only Gaussian noise impact

However, the most impact on channel is the fading problem. With the impact of Gaussian noise and multi-path fading, there are many error bits at receiver 
Research, Development and Application on Information and Communication Technology

and we can not recognize original data anymore.

Without channel estimation, noise and fading make many error bits, at receiver when compared with original data. That is why channel estimation is very important in mobile communication.

B. Channel estimation with comb-type pilot training structure and interpolation algorithms:

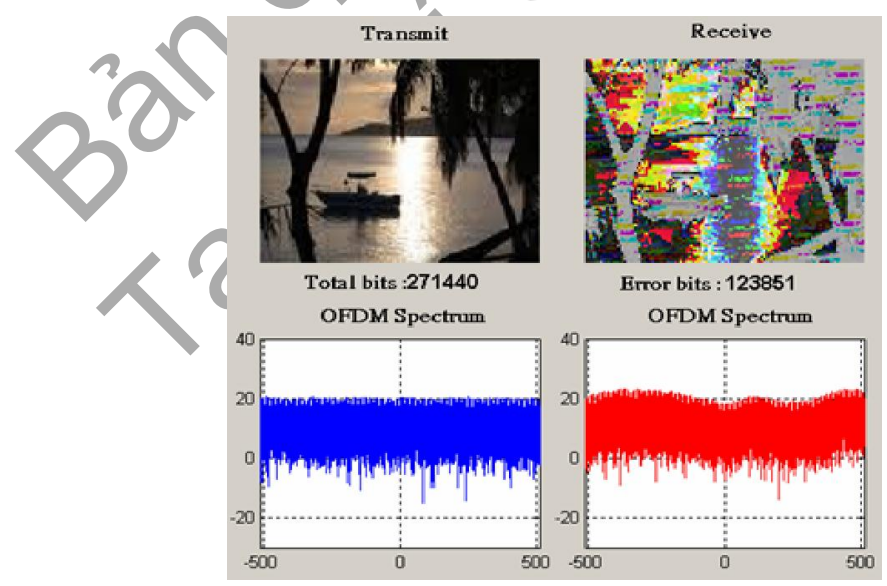

Figure 10. Simulation without channel estimation, $S N R=20 \mathrm{~dB}, 4-Q A M$ with impact of Gaussian noise and Rayleigh fading (indoor enviroment-velocity $1 \mathrm{~km} / \mathrm{h}$ )

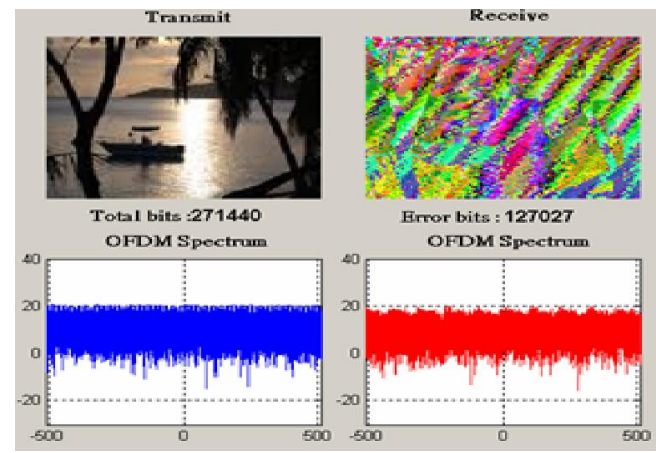

Figure 11. Simulation without channel estimation, $S N R=20 \mathrm{~dB}, 4-Q A M$ with impact of Gaussian noise and Rayleigh fading (vehicular model -velocity $100 \mathrm{~km} / \mathrm{h}$ )

In the above simulations, we proved that channel estimation is necessary in mobile communication and in this part we will prove how channel estimation rejects noise and fading. In channel estimation with comb-type pilot training structure, we need to use an interpolation algorithm to interpolate data at sub- carrier frequencies. In the following simulation, we can see the results with channel estimation using linear interpolation and cubic spline interpolation. The modulation scheme is 4-QAM, the channel is impacted by Gaussian noise and Rayleigh fading with pedestrian enviroment, velocity of $5 \mathrm{~km} / \mathrm{h}$

\section{BER simulation of interpolation algorithms in mobile WiMAX standard.}

We simulate in three modulation schemes in mobile WiMAX standard: 4-QAM (QPSK), 16-QAM and 64-QAM. We transmit an image file (271440 bits) five times. The result is the average of transmission for five times and the numbers of bits is long enough to run simulations. This helps us to have correct BER simulation and to limit wrong conclusion.

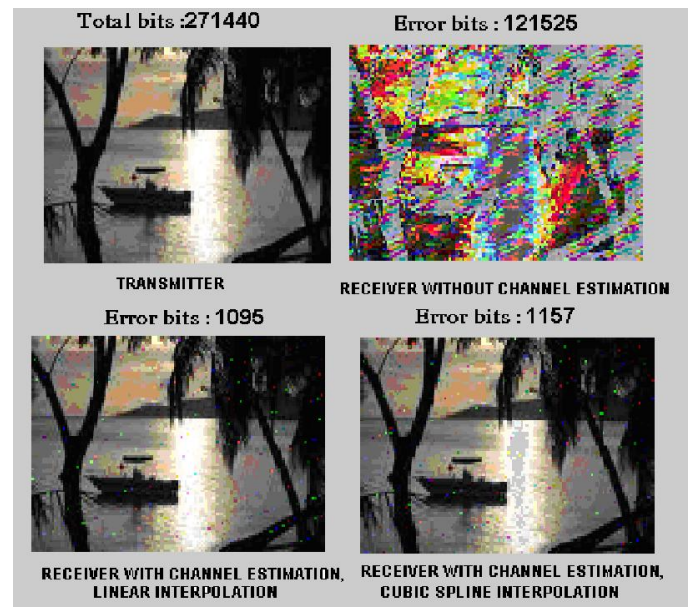

Figure 12. Comparison between receiving data with channel estimation and without channel estimation

\section{1) 4-QAM modulation:}

The simulation results show that the linear interpolation has the lowest SNR for all 3 environments (indoor, pedestrian and vehicular).

4-QAM modulation is used whenever receivers have low SNR and low power e.g. when they are far away from transmitters. Among 3 modulation schemes (4-QAM, 16-QAM, 64-QAM), this modulation shows the lowest perfomance (2 bit/symbol) but has the least level of noise and multi- 
path problem impacts. That nearest neighbor interpolation which has still accepted results and better results than spline cubic (Figure 13) proved Gaussian noise and the multi-path fading impact the system complicatedly with this modulation. As the result, the channel response estimation of radio channels with linear interpolation proved the best results in simulation. Linear interpolation with very low complexity is suggested to be used in this modulation.

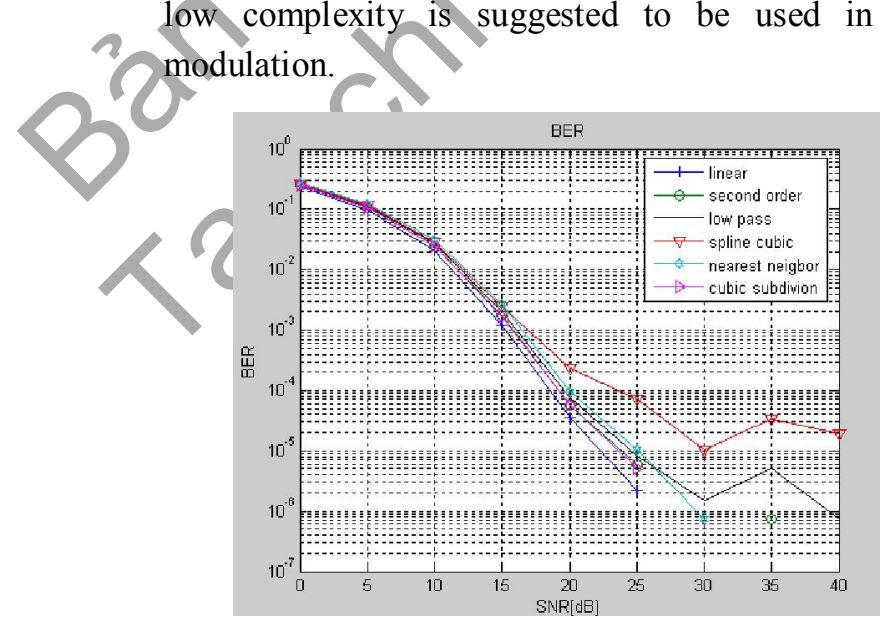

Figure 13. Indoor model with mobile speed of $1 \mathrm{~km} / \mathrm{h}$, 4-QAM modulation

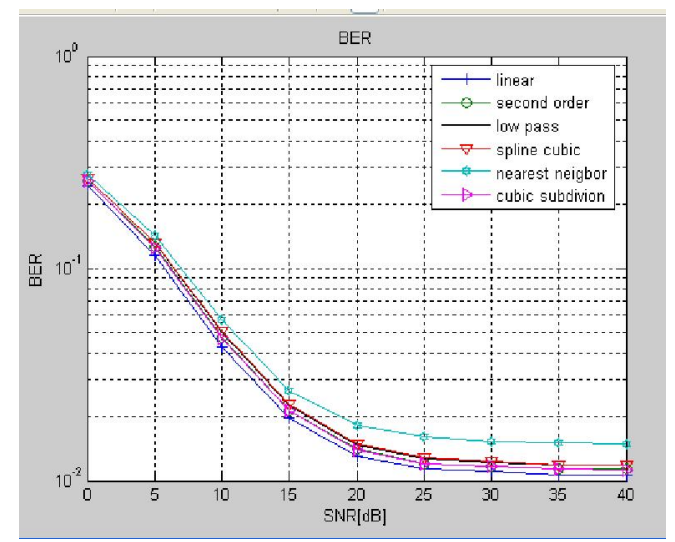

Figure 14. Pedestrian model with mobile speed of 5 $\mathrm{km} / \mathrm{h}, 4-Q A M$ modulation

\section{2) 16-QAM modulation:}

The simulation results show that linear interpolation has the best results at all simulation environments. The second algorithm has the good result is cubic sub-division.

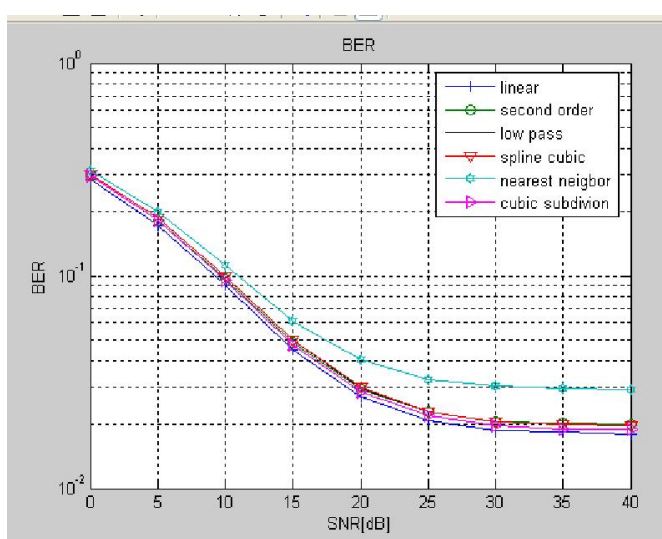

Figure 15. Vehicular model with mobile speed of $100 \mathrm{~km} / \mathrm{h},-4$ - QAM modulation

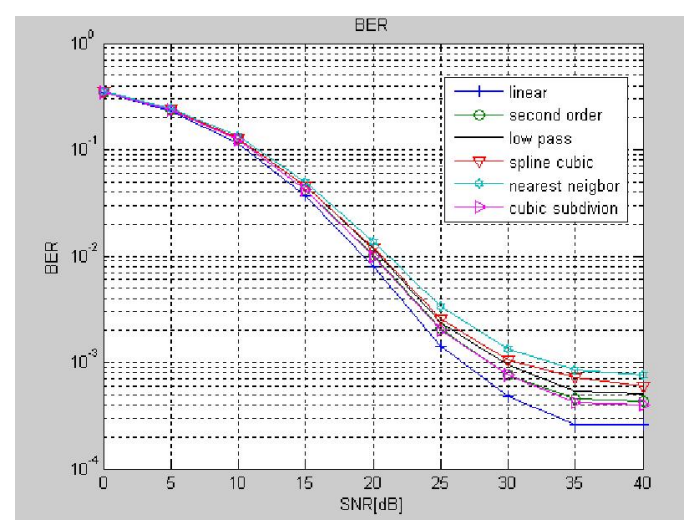

Figure 16. Indoor model with mobile speed of $1 \mathrm{~km} / \mathrm{h}$, 16-QAM modulation

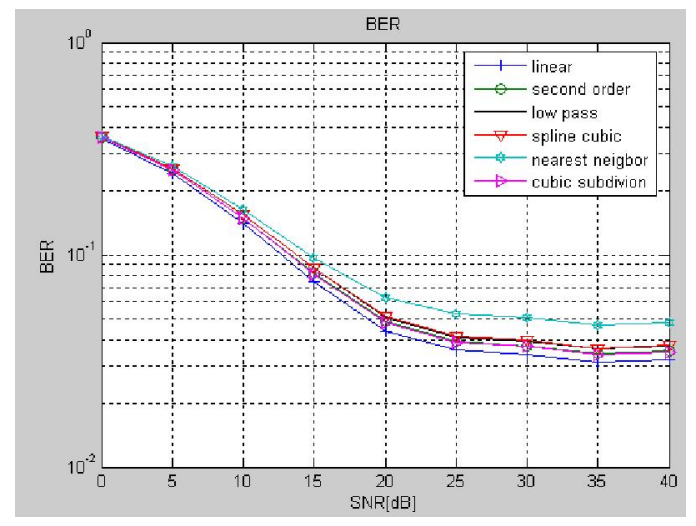

Figure 17. Pedestrian model with mobile speed of $5 \mathrm{~km} / \mathrm{h}, 16-Q A M$ modulation 
Research, Development and Application on Information and Communication Technology

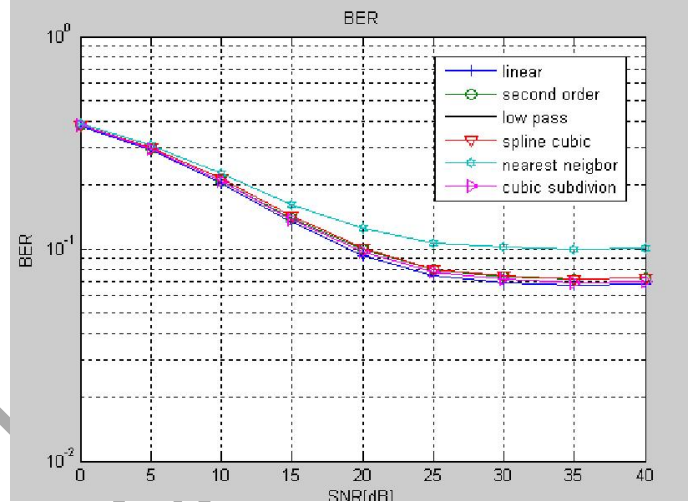

Figure 18. Vehicular model with mobile speed of $100 \mathrm{~km} / \mathrm{h}, 16-Q A M$ modulation

The system uses this modulation when receivers have good SNR and good environment condition. Compared with 4-QAM, this modulation show better throughput (4 bits/symbol) but the BER performance is worse than 4-QAM modulation. This means Gaussian noise and Rayleigh fading make impact much on radio channels and the channels change complicatedly. Bit errors are more than 4-QAM scheme. Linear interpolation still works the best cubic sub-division is the second one.

3) 64-QAM modulation:

The simulation results show that linear interpolation works the best in indoor and pedestrian model, cubic subdivision has the best performance in vehicular environment.

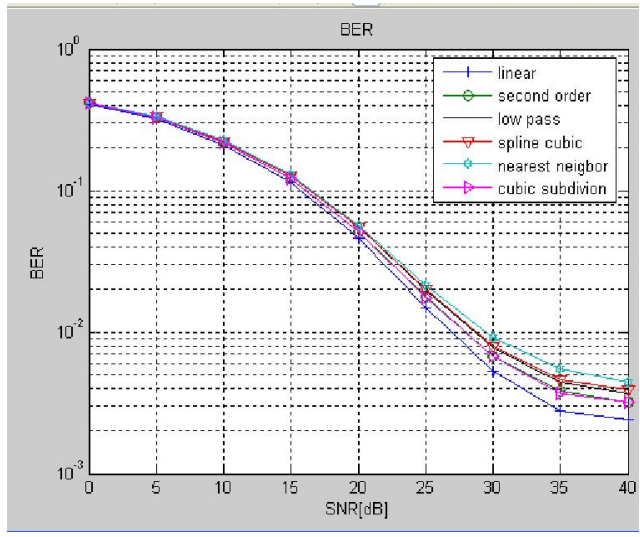

Figure 19. Indoor model with mobile speed of $1 \mathrm{~km} / \mathrm{h}$, 64-QAM modulation

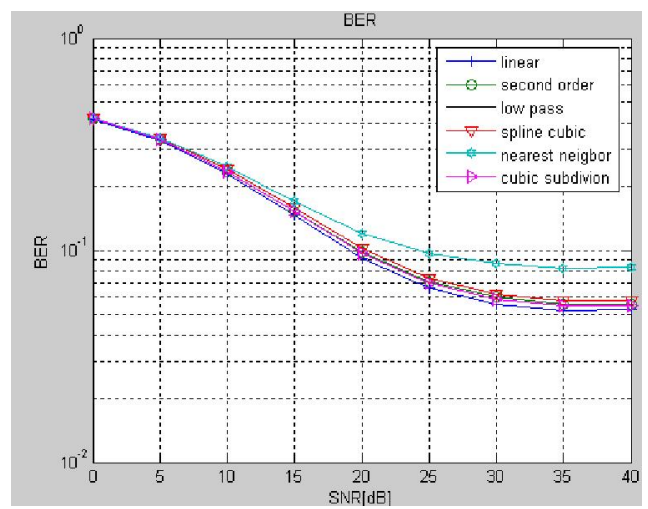

Figure 20. Pedestrian model with mobile speed of $5 \mathrm{~km} / \mathrm{h}$, 64- QAM modulation

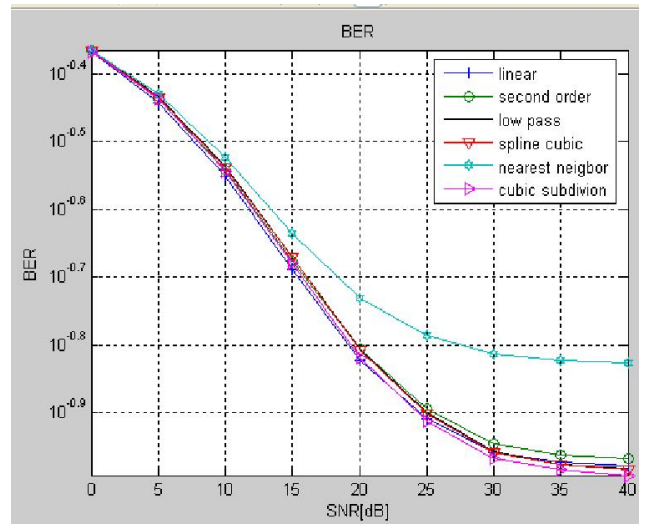

Figure 21. Vehicular model with mobile speed of $100 \mathrm{~km} / \mathrm{h}, 64-Q A M$ modulation

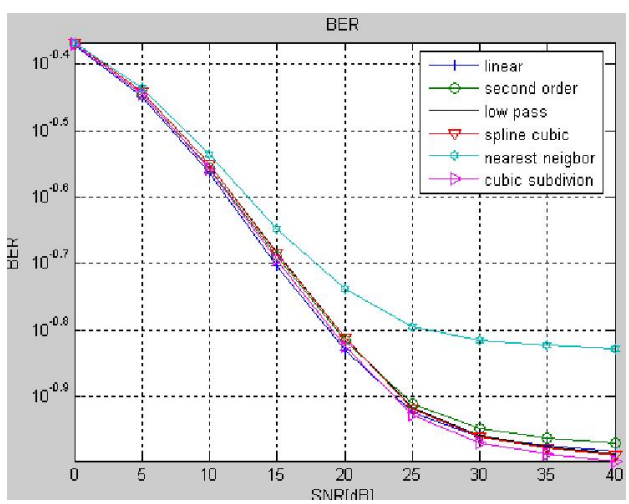

Figure 22. Vehicular model with mobile speed of $80 \mathrm{~km} / \mathrm{h}, 64-Q A M$ modulation

We continue simulation with 64-QAM scheme for vehicular environment to show that cubic sub-division is better choice than linear in this case. The below 
results prove that cubic sub-division is the best one is this model. With speed of $60 \mathrm{~km} / \mathrm{h}$ and $80 \mathrm{~km} / \mathrm{h}$, cubic sub-division has the best performance in simulation.

The system uses 64-QAM modulation when receivers have very good SNR, for instance, MS is very near transmitters with very high power. This modulation gives the best throughput ( 6 bits/symbol) but noise and multi path fading make the system have the most error bits among 3 simulation schemes. Simple and low order interpolation algorithms such as nearest neighbor and linear interpolation can not have better results than higher order interpolation in fast fading channel environment with high velocity. With heavily impacts of Gaussian noise and fading, high order interpolations are feasible in this case and cubic subdivision is proved to be the best choice in simulations.

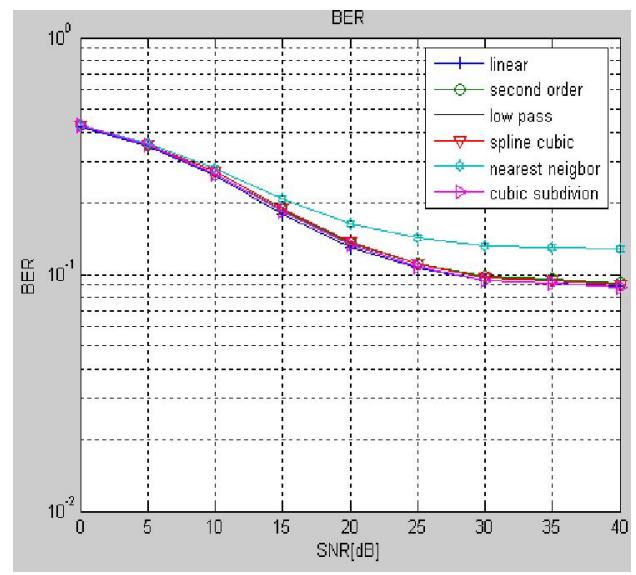

Figure 23. Vehicular model with mobile speed of $60 \mathrm{~km} / \mathrm{h}, 64-Q A M$ modulation

\section{CONCLUSION}

The paper analyzed channel interpolation algorithms in the LS estimation algorithm, the pilot is comb-type, the parameters used in the paper are simulated with standard IEEE 802.16E. The paper also analyzed the relation between impacts of Noise and fading and interpolation algorithms. We simulated mobile WiMAX system at frequency $2.5 \mathrm{GHz}$ and bandwidth $20 \mathrm{MHz}$. The simulation results proved that in 4-QAM and 16-QAM modulation, the linear interpolation has the best results in 3 models: indoor, pedestrian and vehicular. In 64-QAM modulation, cubic sub-division interpolation has the best results in vehicular model.

The WiMAX system has low order modulations such as 4-QAM whenever receivers have low SNR and linear interpolation is recommended to for the estimation channel system with comb-type pilot because this interpolation show the best results . When receivers have good SNR, the WiMAX system has higher order modulation such as 16-QAM and 64QAM. In 64-QAM scheme, cubic sub-division interpolation, which show good results in vehicular model, is suggested to be used in fast fading channel model for vehicular model.

For future researches, we will pay attention to some other algorithms and improve the potential algorithms to have better performance.

\section{REFERENCES}

[1] F. Wang, A. Ghosh, "Mobile WiMAX Systems: Performance and Evolution", IEEE Communications Magazine, vol. 46, no.10, October 2008, pp.41-49.

[2] WiMAX Forum, "WiMAX System Evaluation Methodology", Version 2.1, July 2008.

[3] Muhammad Saad Akram, "Pilot-based Channel Estimation in OFDM", Systems Master Thesis, Nokia Denmark, 2007.

[4] Yushi Shen and Ed Martinez, "WiMAX channel Estimation: algorithm and Implementations", Document number AN3429., Austin Texas ,07/2007.

[5] Daniel Larrson, "Analysis of channel estimation methods for OFDMA", Master of Science Thesis, Stockholm, Sweden 2006-12-19.

[6] J.-J. van de Beek, O. Edfors, M. Sandell, S. K. Wilson, and P. O. Borjesson, "On channel estimation in OFDM systems", in Proc. IEEE $45^{\text {th }}$ Vehicular Technology Conf., Chicago, IL, Jul. 1995.

[7] Meng-Han Hsieh and Che-Ho Wei, "channel estimation for ofdm systems based on comb-type pilot arrangement in frequency selective fading channels", IEEE Transactions on Consumer Electronics, Vol. 44, No. 1, February 1998.

[8] J.-J. van de Beek, O. Edfors, M. Sandell, S. K. Wilson, and P. O. Borjesson, "On channel estimation in 
Research, Development and Application on Information and Communication Technology

OFDM systems", in Proc. IEEE $45^{\text {th }}$ Vehicular Technology Conf., Chicago, IL, Jul. 1995.

[9] "Channel models for Fixed Wireless Application," IEEE 802.16.3c - 01/29r4, 16 July, 2001

[10] Rebeca M.Colda, Tudor Palade, "Transmission Performance Evaluation of Mobile WiMAX Pedestrian Environments" 17th Telecommunications forum TELFOR, Serbia, Belgrade, November 24-26, 2009.

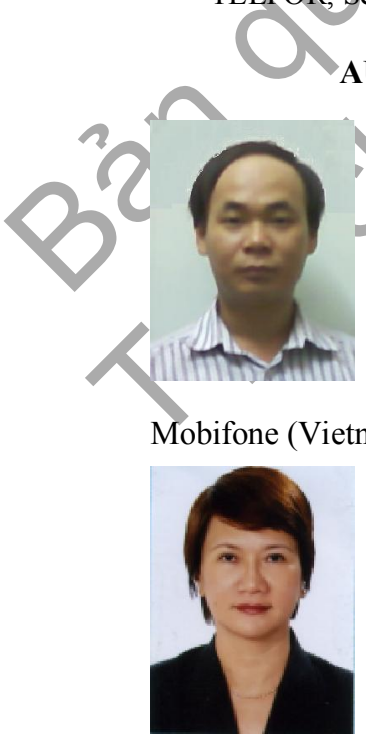

\section{AUTHORS' BIOGRAPHIES}

Nguyen Duc Quang graduated university in 2006 and received Master degree at HCMC University of Technology in 2008, being currently $\mathrm{PhD}$ student at Telecommuniation Department in the same university. Research areas: WiMAX, estimation channel, communication system. $\mathrm{He}$ is working for Mobifone (Vietnam Mobile Service Company).

Pham Hong Lien graduated university in 1979 and received her doctorate at the University of Slovakia in 1993. She was promoted to associate professor positions in 2006.

She is vice dean of Electrics-Electronics Department at University of Technical Education, Ho Chi Minh city, Vietnam. Research areas: Telecommunications networks (PBX, data communication networks, mobile networks, WDM optical networks, SDH...) Computer Networks (ATM, MPLS, NGN ..)

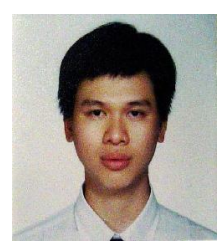

Luu Thanh Tra graduated university in 2001 and received master degree in engineering in 2008 at Pierre Marie Curie University, Paris, France, specialized in networking. He received doctoral degree at the Ecole Nationale Supérieure Telecommunications (ENST), Paris Specialized in Telecommunication Network. He is a department head of Networking Management, University of Technology, Vietnam National University, Ho Chi Minh city, Vietnam.

Research areas: Computer networks, telecommunications networks and data transmission. 